terschied in der Wellenlänge der Farbbande nimmt mit steigendem Elektronendonator-Charakter des Substituenten zu. Zwischen den Verbindungen mit $\mathrm{R}=-\mathrm{OCH}_{3}$ und $\mathrm{R}=-\mathrm{Br}$ bzw. $-\mathrm{Cl}$ beträgt der Wellenlängenunterschied der Farbbande bei 4-Substitution $44 \mathrm{~nm}$ und ist somit mit dem $4 \lambda$ von 3 bei 5-Substitution vergleichbar. Die Frequenz der $v(\mathrm{C}=\mathrm{C})$-Absorption liegt bei ElektronendonatorSubstitution in 4-Stellung bei $\mathbf{1}$ höher als in 5-Stellung. Für die $v(\mathrm{C}=\mathrm{O})$-Absorption wird der inverse Frequenzgang beobachtet. Der stärkere Substituenteneinfluß bei 4-Substitution gegenüber 5-Substitution ist darauf zurückzuführen, daß erstere $p$-ständig und letztere $m$-ständig zum Pyryliumsystemteil erfolgt.

Für $\mathrm{R}=-\mathrm{H}$ und $-\mathrm{CH}_{3}$ nimmt die Brückenbindung der $o$-Hydroxy-Gruppe von $\mathbf{1}$ über $\mathbf{2}$ nach $\mathbf{3}$ zu. In der gleichen Reihenfolge sinkt die $v(\mathrm{C} \cdots \mathrm{O})$ Frequenz ab und die $v(\mathrm{Cl}-\mathrm{O})$-Frequenz steigt an. Die Kopplung der o-Hydroxy-Gruppe mit dem Pyryliumsauerstoff über das Anion ist demnach bei $\mathbf{3}$ am größten. Die Kopplung nimmt dann über 2 nach 1 wahrscheinlich deswegen ab, weil in dieser Richtung das Gesamtsystem planarer wird und dadurch der Substituenteneinfluß sich auf das Gesamtsystem verteilt. Für Substituenten mit stärkerem mesomeren Substituenteneffekt wird die obige Reihenfolge derart abgeändert, daß die Planarität des Styrylpyryliumsystems von $\mathbf{2}$ über $\mathbf{3}$ nach $\mathbf{1}$ ansteigt.

Abschließend sei dem Bundesministerium für wissenschaftliche Forschung für die Förderung der Untersuchungen und den Herren Dr. C. Schiele und G. PAal für die Darstellung der $o$-Hydroxystyrylpyryliumperchlorate gedankt.

\title{
Zum Verhalten von Spirobipyranen und ihren farbigen Formen
}

\author{
Cajetan Schiele* \\ AEG-Telefunken Forschungsinstitut Ulm/Donau \\ (Z. Naturforschg. 24 b, 976-988 [1969]; eingegangen am 7. März 1969)
}

\begin{abstract}
Ten types of symmetrically and asymmetrically synthesized spirobipyrans and their basic $o$-hydroxyarylvinylpyryliumsalts were investigated. A clearer understanding was achieved of the mechanisms involved in the processes from ring opening to the formation of colored species. The investigations were concerned with unsubstituted asymmetric spirobipyrans as well as those substituted in a-position to the spiro-C-atom in one of the double bonds of the pyran ring-systems. The purpose was to understand the behaviour of the compounds by measuring changes in their structural parameters.
\end{abstract}

Spirobipyrane und Trimethylindolinospiropyrane werden bis heute in der Literatur ohne Differenzierung behandelt, deshalb werden auch zum Verhalten von Spiropyranen recht unterschiedliche Aussagen gemacht ${ }^{1-4}$. Die meisten Autoren schlagen eine Merocyaninformulierung bei der Bildung farbiger Formen vor, Dilthey ${ }^{5}$ und Wizinger ${ }^{6}$ hingegen formulieren eine ionische Dissoziation und begründeten diese durch eine chemische Beweisführung. Keine dieser Ansichten schien uns jedoch das Verhalten von Spirobipyranen klar genug zu interpretieren:

* Neue Anschrift: Chemische Landesuntersuchungsanstalt 7 Stuttgart, Kienestr. 18

1 G. KortüM, Optische Anregung organischer Systeme, Verlag Chemie. Weinheim 1966, Seite 468.

2 M. Pestemer, Optische Anregung organischer Systeme, Verlag Chemie. Weinheim 1966. Seite 481.

${ }^{3}$ E. Fischer, Fortschritte der chemischen Forschung, Band $7 / 3$, Seite 626 .
Eine Merocyaninformulierung ist unbefriedigend, weil sich bei ihr die Lage der Absorptionsmaxima durch Lösungsmittel unterschiedlicher DK verschieben lassen sollte, was aber bei Raumtemperatur bei Spirobipyranen nicht der Fall ist. Es wurde zudem von Hünig und Rosenthal ${ }^{7}$ gezeigt, daß bei Merocyaninen, bei denen der Aromat in ein konjugiertes System eingeschaltet ist, deren Verhalten außer von der Natur der Endgruppen in entscheidendem Maße von der Entaromatisierungs-Tendenz des Aromaten abhängt.

${ }^{4}$ E. B. KNott, J. chem. Soc. [London] 1951, 3038.

5 W. Dilthey, C. Berres, E. Hölterhoff u. H. Wübken, J. prakt. Chem. 114, 179 [1926].

6 R. Wizinger u. H. Wenning, Helv. chim. Acta 23, 247 [1940].

7 S. Hünig u. O. Rosenthal, Liebigs Ann. Chem. 592, 161 [1955]. 
Die Dilthey sche Formulierung stellt trotz Beweisführung nicht völlig zufrieden, weil sich mit ihr zwar bei ein und derselben Verbindung verschiedene Absorptionsmaxima erklären lassen, $o$-Hydroxyarylvinylpyrylium-Salze verschiedener Strukturen jedoch weder präparativ dargestellt noch aus spektroskopischen Befunden sicher nachgewiesen werden konnten.

Wie bei den vor kurzem analysierten Trimethylindolinospiropyranen ${ }^{8}$ wurde deshalb das Entstehen der farbigen Formen von Spirobipyranen experimentell untersucht. Geht man hierbei der chemischen Beweisführung von DilTHEY ${ }^{5}$ folgend davon aus, da $ß$ als Normalprozeß für das Entstehen einer farbigen Form eine ionische intramolekulare Dissoziation anzunehmen ist, so ergeben sich bei Spirobipyranen für das Molekül die in Formel 1 skizzierten Möglichkeiten. Alle bei Thermo-, Solvato- und Photochromie, wie auch bei der Einwirkung von Säuren zu beobachtenden farbigen Formen sollten demnach auf dem Vorhandensein der in Formel 1 skizzierten nicht protonierten oder protonierten intramolekular dissoziierten Strukturen B und C beruhen. Das Vorliegen anderer (sterisch mehr behinderter) Strukturen ist bei Raumtemperatur oder erhöhten Temperaturen kaum anzunehmen.

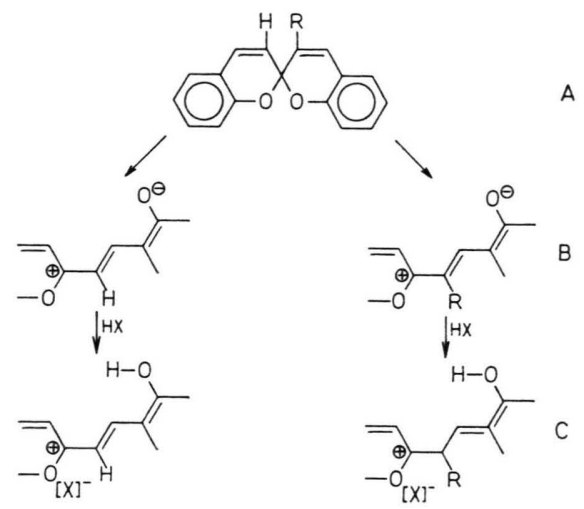

Formel 1

Um einen Überblick über das Verhalten von Spirobipyranen zu gewinnen, wurden die Daten folgender substituierter und unsubstituierter Verbindungen der Typen $\mathbf{1}$ - $\mathbf{1 0}$ erfaßt:

$1 \quad 2.2^{\prime}$-Spirobi[2H-1-benzopyrane],

2 Spiro[2H-1-benzopyran-2.2'- $\left(2^{\prime} \mathrm{H}\right)$ naphtho [1.2-b] pyrane],

8 C. Schiele u. G. Arnold, Z. Naturforschg. 23 b, 628 [1968].
3 Spiro[2H-1-benzopyran-2.3'- $\left(3^{\prime} \mathrm{H}\right)$ naphtho

[2.1-b] pyrane],

4 Spiro[2H-1-benzopyran-2.2' $-\left(2^{\prime}\right.$-phenanthreno

[2.1-b] pyran],

5 Spiro[2H-1-benzopyran-2.3'- $\left(3^{\prime} \mathrm{H}\right)$ anthraceno

[2.1-b] pyran],

$62.2^{\prime}$-Spirobi[2H-naphtho[1.2-b] pyrane],

7 Spiro[2H-naphtho[1.2-b]pyran-2.3'- $\left(3^{\prime} \mathrm{H}\right)$ naphtho [2.1-b] pyrane],

$83.3^{\prime}$-Spirobi[3H-naphtho[2.1-b] pyrane],

9 Spiro $\left[3 \mathrm{H}\right.$-naphtho $[2.1-\mathrm{b}]$ pyran-3.2' $-\left(2^{\prime} \mathrm{H}\right)$ phenanthreno[2.1-b] pyran],

10 Spiro[3H-naphtho[2.1-b] pyran-3.3'- $\left(3^{\prime} \mathrm{H}\right)$ anthraceno[2.1-b] pyran].

Von diesen zeigten die am Pyranring unsubstituierten Verbindungen 3, $\mathbf{4}$ und $\mathbf{5}$ in saurer Lösung einen deutlichen Gang der Lage ihrer längstwelligen Absorptionsbande, der bei 7, 9 und 10 nicht zu beobachten war. Da allerdings im Gegensatz zu Trimethylindolinospiropyranen dieser Gang nicht im Verlaufe einer Hell-Dunkel-Reaktion ${ }^{8}$ zu beobachten war, erhob sich die Frage, ob die Frequenzverschie-

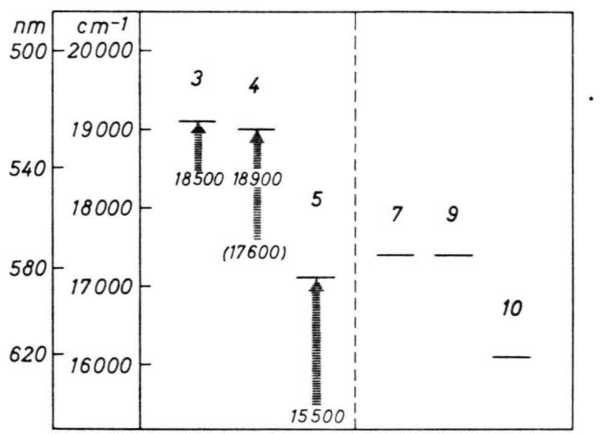

Tab. 1. Lage der längstwelligen Absorptionsbande bei asymmetrischen, unsubstituierten Spirobipyranen in 0,1-m. methanolischer $\mathrm{HClO}_{4}$.

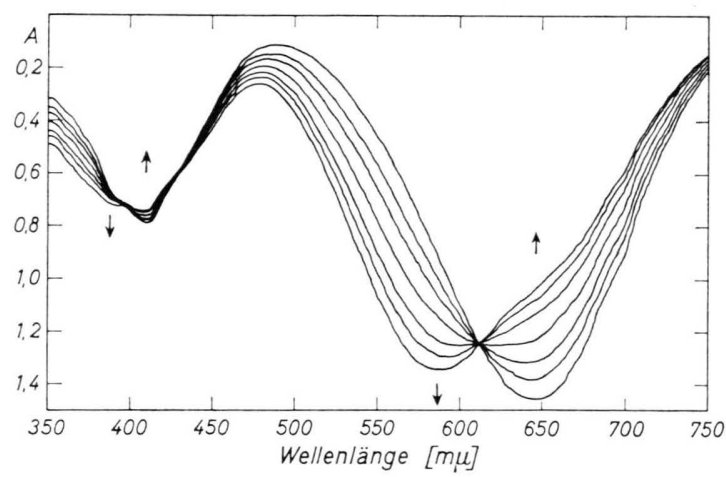

Abb. 1. Gang der längstwelligen Absorptionsbande beim Spiro [2H-1-benzopyran-2.3'- $\left(3^{\prime} \mathrm{H}\right)$ anthraceno [2.1-b]pyran] 5 in $0,1-m$. methanolischer $\mathrm{HClO}_{4}$. Registrieren des Spektrums alle 3 Minuten. 


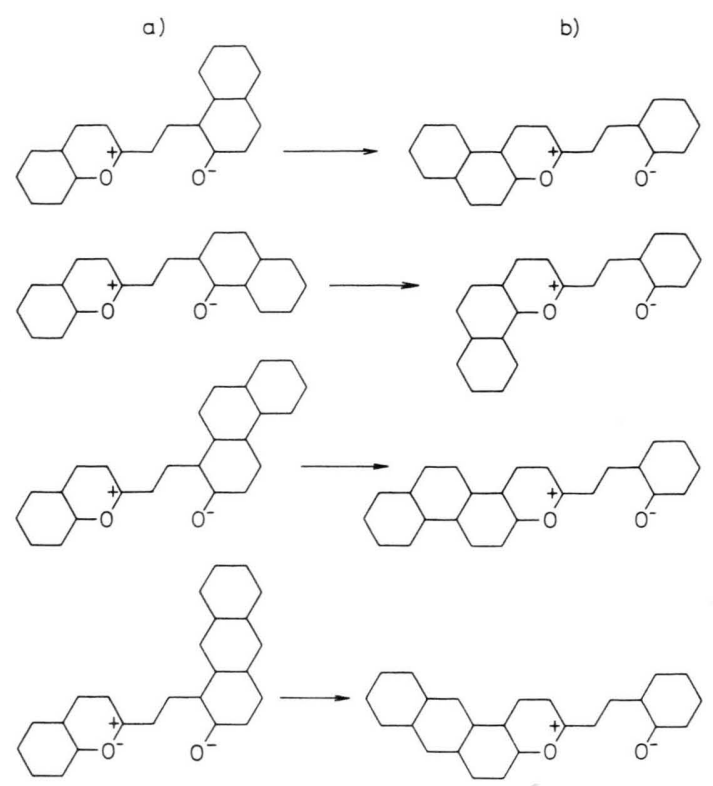

Formel 2 bung nicht einer Isomerisierung des Systems (Formel 2) auf Grund einer Verschiebung des Dissoziationsgleichgewichtes der zunächst nach Typ a dissoziierten Molekel durch Umwandlung in eine Molekel vom Typ b zuzuordnen sein sollte. Der isosbestische Punkt (Abb. 1) beweist, daß bei Beginn und am Ende der spektroskopisch verfolgten Umlagerung strukturell verschiedene Individuen vorliegen.

Um ein möglichst umfassendes Material zur Interpretation des Verhaltens von Spirobipyranen heranzuziehen, wurden zunächst die aus eigenen Messungen und der vorliegenden Literatur erhaltenen Daten der farbigen Formen von Spirobipyranen zusammengefaßt. Einen Überblick über die sowohl beim Auftreten von Thermochromie wie auch beim Einwirken von Säuren und UV-Bestrahlung in Lösungen verschiedener Temperaturen zu beobachtenden Absorptionsmaxima liefert Tab. 2.

Das so aus Literaturwerten ${ }^{9-14}$ und eigenen Messungen ${ }^{15-19}$ erhaltene Bild ist nicht eindeutig, ob-

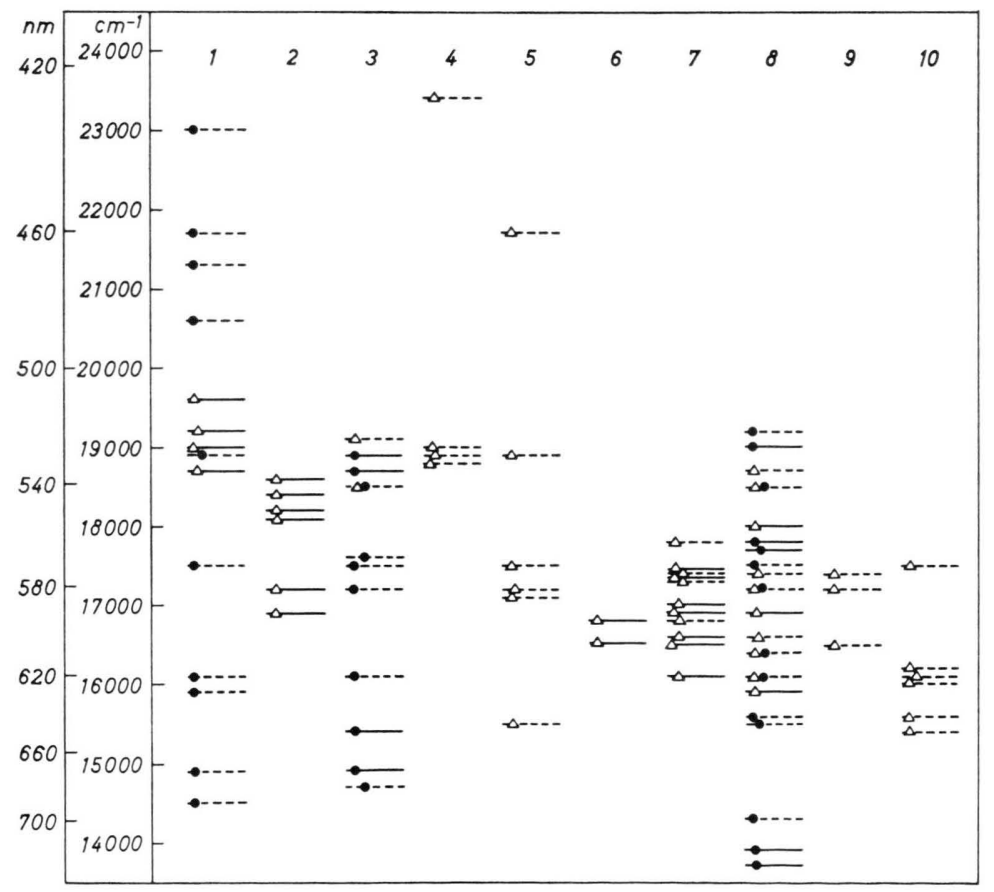

Tab. 2. Nach Ringöffnung beobachtete Absorptionsmaxima am Pyranring substituierter (-) und unsubstituierter (...) Spirobipyrane der Typen $\mathbf{1}-\mathbf{1 0}$. $\triangle=$ Eigenmessung, $\bullet=$ Messung anderer Autoren.

9 Y. Hirshberg u. E. Fischer, J. chem. Soc. [London] 1954. 297.

10 E. Fischer u. Y. Hirshberg, J. chem. Soc. [London] 1952 , 4522.

11 R. Heiligman-Rim, Y. Hirshberg u. E. Fischer, J. chem. Soc. [London] 1961, 156.

12 Y. Hirshberg. J. chem. Physics 27, 758 [1957].

13 Y. Hirshberg u. E. Fischer, J. chem. Soc. [London] 1954. 3129 .
14 O. Chaudé, Cahiers de Physique 50, 17: 51. 6: 52, 3 [1954].

15 C. Schiele, A. Wilhelm, D. Hendriks, M. Sterec u. G. PAAL, Tetrahedron [London] 24, 5029 [1968].

16 C. Schiele, G. Paal, G. Arnold u. M. Stepec. Tetrahedron [London] 24, 5037 [1968].

17 Unveröffentlichte Arbeiten von M. STEPEC.

18 Unveröffentlichte Arbeiten von K. Halfar.

19 Unveröffentlichte Arbeiten von B. KÜBLER. 
wohl sich die Auswahl der Daten der an Verbindungen der Typen $\mathbf{1}$ - $\mathbf{1 0}$ gemachten Beobachtungen auf die unsubstituierten oder lediglich am Pyranring substituierten Verbindungen beschränkt. Als Auswirkung einer derartigen Substitution in $\alpha$-Stellung zum Spiro-C-Atom ist im wesentlichen eine nur auf sterische Gründe zurückzuführende Beinflussung des Verhaltens sowohl der Spirobipyrane wie auch der ihnen zugrunde liegenden (und aus ihnen wieder erhältlichen) $o$-Hydroxyarylvinylpyrylium-Salze anzunehmen. Sie war also im weiteren getrennt von der auf Aromatensubstitution zurückzuführenden Beeinflussung zu untersuchen.

\section{Auswertung der Daten von o-Hydroxyarylvinyl- pyrylium-Salzen}

Messungen an $o$-Hydroxyarylvinylpyrylium-Salzen ${ }^{15,16}$ ergaben folgende Gesetzmäßigkeiten:

1. Die $v(\mathrm{C}=\mathrm{O})$-Frequenz unsubstituierter $o$-Hydroxyarylvinylpyrylium-Salze weicht in charakteristischer Weise von der vinylsubstituierter $o$-Hydroxyarylvinylpyrylium-Salze ab, wie mit den nachfolgend angeführten Beispielen gezeigt wird.

Pyryliumperchlorat $v(\mathrm{C} \cdots \mathrm{O})$ in $\mathrm{cm}^{-1}$

3 -[o-Hydroxystyryl] naphtho[2.1-b]1527

3 -[(1-Hydroxy-2-naphthyl) vinyl] naphtho[2.1-b]- 1521

3 -[(2-Hydroxy-1-naphthyl) vinyl] naphtho[2.1-b] - 1521 3 -[ $\beta$-n-Amyl-o-hydroxystyryl] naphtho[2.l-b] - $\quad 1548$

3

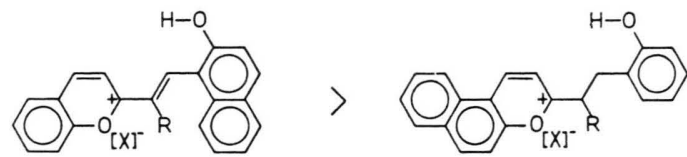

3-[ $\beta$-n-Amyl-(1-hydroxy-2-naphthyl) vinyl] naphtho[2.1-b]

3-[ $\beta$-n-Amyl-(2-hydroxy-1-naphthyl) vinyl] naphtho[2.1-b]-

2. Die Lage des Absorptionsmaximums wird im wesentlichen vom phenolischen Teil des Moleküls bestimmt. Die bei unseren strukturspezifischen Synthesen untersuchten Verbindungstypen $\mathbf{1}-\mathbf{3}$ und 6 - 8 zeigen die in Formel 3 skizzierten Lagen der langwelligsten Absorptionsbanden in 0,1-m-methanolischer $\mathrm{HClO}_{4}{ }^{15,16}$. Für die substitutionsabhängige Lage der Maxima gilt generell: Unsubstituierte $<$ alkylvinylsubstituierte < arylvinylsubstituierte Verbindung.

3. Die bei $o$-Hydroxystyrylpyrylium-Salzen bei Substitution des Phenylrestes zu beobachtende Verschiebung der Absorptionsmaxima zeigt denselben Gang, wie er bei analog substituierten Trimethylindolinospiropyranen ${ }^{8}$ beobachtet wurde.

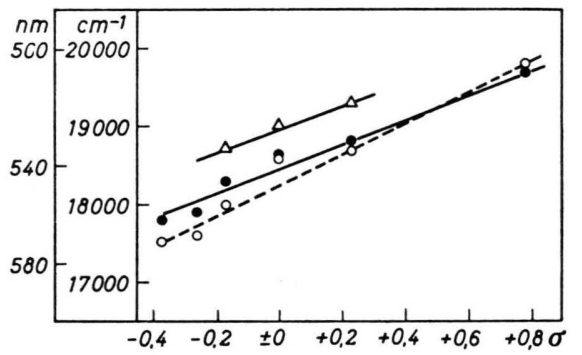

Tab. 3. Gang der langwelligsten Absorptionsbande mit der Substitution des Phenylrestes para zur OH-Gruppe bei 3-[ $\beta$ - $n$ Amyl - $o$-hydroxystyryl] naphtho [2.1 - b] pyryliumperchloraten (๑), 2 -[ $\beta$-n-Amyl-o-hydroxystyryl] naphtho [1.2-b]pyryliumperchloraten (o) und 3-(o-Hydroxystyryl) naphtho[2.1-b] pyry3 liumperchloraten $(\triangle)$. Auftragung gegen die $\sigma$-Konstante des Phenylsubstituenten.

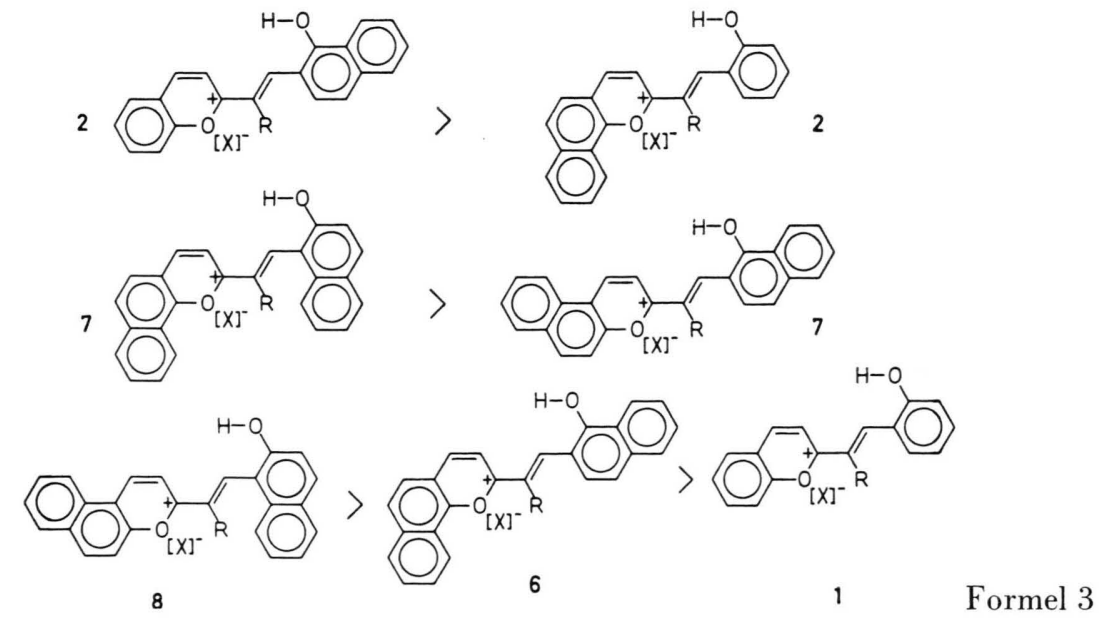




\begin{tabular}{|c|c|c|c|c|c|c|c|c|}
\hline $\begin{array}{l}\text { Phenyl- } \\
\text { sub- } \\
\text { stituent }\end{array}$ & $\sigma_{\mathrm{I}}$ & $\sigma_{\mathrm{M}}$ & $v(\mathrm{OH})$ & $v(\mathrm{C}=\mathrm{C})$ & $v(\mathrm{C}=\mathrm{O})$ & $v(\mathrm{Cl}-\mathrm{O})$ & $v(\mathrm{C}-\mathrm{O})$ & $\begin{array}{c}\log \varepsilon \text { der } \\
\text { langwelligsten } \\
\text { Bande } 15,16\end{array}$ \\
\hline \multicolumn{9}{|c|}{ 3-[ $\beta$-n-Amyl-o-hydroxystyryl]-naphtho[2.1-b]pryryliumperchlorate } \\
\hline $\mathrm{CH}_{3-}^{-}$ & $-0,05$ & & 3290 & 1608 & 1547 & 626,1097 & 1049 & 4,60 \\
\hline $\mathrm{H}-$ & \pm 0 & & 3260,3200 & 1615 & 1548 & 624,1112 & 1050,1040 & 4,57 \\
\hline $\mathrm{Br}-$ & $\overline{+} 0,45$ & & 3195 & 1607 & 1544 & 622,1105 & 1053 & 4,38 \\
\hline \multirow{3}{*}{\multicolumn{2}{|c|}{$\begin{array}{l}\mathrm{HO}- \\
\mathrm{CH}_{3} \mathrm{O}- \\
\mathrm{NO}_{2-}\end{array}$}} & $-0,61$ & 3375 & 1616 & 1545 & 628,1117 & 1065 & 4,45 \\
\hline & & $-0,50$ & 3245 & 1607 & 1546 & 623,1117 & 1050,1034 & 4,46 \\
\hline & & $+0,15$ & & 1617 & 1554 & 624,1083 & & 4,64 \\
\hline \multicolumn{9}{|c|}{ 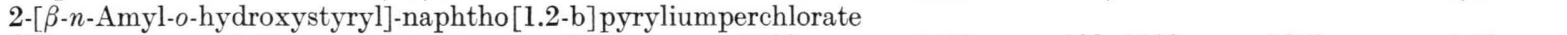 } \\
\hline $\mathrm{CH}_{3-}$ & $-0,05$ & & 3278 & 1632 & 1546 & 623,1103 & 1051 & 4,42 \\
\hline $\mathrm{H}-$ & \pm 0 & & 3215 & 1633 & 1545 & 622,1109 & 1050,1034 & 4,40 \\
\hline $\mathrm{Br}-$ & $\mp 0,45$ & & 3145 & 1628 & 1541 & 622,1105 & 1029 & 4,30 \\
\hline \multirow{3}{*}{\multicolumn{2}{|c|}{$\begin{array}{l}\mathrm{HO}- \\
\mathrm{CH}_{3} \\
\mathrm{NO}_{2}-\end{array}$}} & $-0,61$ & 3395 & 1633 & 1544 & 625,1089 & 1062 & 4,26 \\
\hline & & $-0,50$ & 3326 & 1632 & 1543 & 625,1105 & 1062,1036 & 4,29 \\
\hline & & $+0,15$ & & 1630 & 1553 & 624,1085 & & 4,32 \\
\hline
\end{tabular}

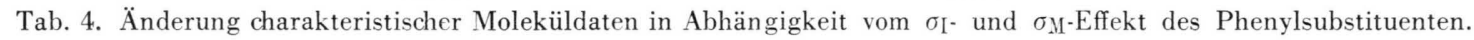

Wie Tab. 4 zeigt, sind die Änderungen der Eigenschaften solcher $o$-Hydroxystyrylpyrylium-Salze dabei auf den Einfluß definierbarer Substituenteneffekte zurückzuführen, es lassen sich Unterschiede in der Einwirkung der einzelnen Substituenten auf Teilstücke des Systems erkennen.

Beim Vergleich der aus vinlysubstituierten Spirobipyranen in saurer Lösung entstehenden farbigen Formen mit den den Spiranen zugrunde liegenden $o$-Hydroxyarylvinylpyrylium-Salzen ${ }^{17}$ zeigte sich schließlich, daß die Absorptionsmaxima der aus den vinylsubstituierten Spirobipyranen in saurer Lösung gebildeten Farbformen denen der o-Hydroxyarylvinylpyrylium-Salze entsprechen. Damit kann bei asymmetrisch aufgebauten Spirobipyranen klar angegeben werden, an welcher Stelle der Ringaufbruch erfolgt. Im Gegensatz zu den unsubstituierten Grundkörpern wurde bei alkyl- und arylvinylsubstituierten Spirobipyranen kein Gang der längstwelligen Absorptionsbande beobachtet. Eine Ausnahme, die später ihre Erklärung findet, bildet dabei jedoch das 2'-Methyl-spiro [2H-1-benzopyran-2.3'-(3'H)naphtho [2.1-b]pyran]. Wir versuchten nun die in Formel 2 formulierte Umlagerung von Spirobenzopyranen zu klären. Als Modellsubstanz wählten wir das aus der Reihe der höher anellierten Spirobipyrane am einfachsten darstellbare Spiro[2H-1-benzopyran-2.3'. ( $\left.3^{\prime} \mathrm{H}\right)$ naphtho[2.1-b] pyran] 3 , bei dem zunächst bei der Aufnahme des Spektrums in saurer Lösung eine Verschiebung des Maximums von 540 nach $520 \mathrm{~nm}$ beobachtet wurde. Die Kontrolle des zeitlichen Ablaufes dieser Verschiebung ließ bei $\mathbf{3}$ jedoch unmittelbar nach $\mathrm{HClO}_{4}$-Zugabe ein anfängliches Maxi- mum bei $570 \mathrm{~nm}$ erkennen; ihre Auswertung führte zu dem Ergebnis, daß die Umlagerung von 3 wie auch seinen Derivaten aus einer Naphtholatstruktur in eine Phenolatstruktur als Reaktion 1. Ordnung mit den folgenden Aktivierungsenergien abläuft ${ }^{18}$ : 6-Methyl-3 = 15,4 $\pm 2,2 \quad \mathrm{Kcal} / \mathrm{Mol}, \quad 6$-Brom-3 = $17,5 \pm 2,5 \mathrm{Kcal} / \mathrm{Mol}$ und $\mathbf{3}=20,0 \pm 3,0 \mathrm{Kcal} / \mathrm{Mol}$.

Zur Interpretation dieser Ergebnisse ist es nötig klarzustellen, welche Vorgänge verfolgt wurden. Intermediär sollten sich (Formel 4) zunächst zwitterionische Phenolat- wie auch Naphtholat-Strukturen ausbilden, aus denen durch Protonenanlagerung die eigentlichen $o$-Hydroxyarylvinylpyrylium-Salze entstehen. Unseren Messungen zufolge stellt sich nun auf Säurezugabe zu Lösungen von Spirodi- $\beta$-naphthopyranen 8 das Gleichgewicht Spiropyran/Pyryliumsalz nahezu spontan ein, es wird auch eine sehr schnell ablaufende Zyklisierung bei $o$-Hydroxyarylvinylpyrylium-Salzen dieses Typs beobachtet. Das Gleichgewicht stellt sich bei Spirodibenzopyranen jedoch erst in einer deutlichen Zeitreaktion ein, analog dazu läuft auch die Zyklisierung von $o$-Hydroxystyrylpyrylium-Salzen dieses Typs langsamer ab. Da demzufolge die Reaktionsschritte $A \underset{1 \mathrm{a}}{\stackrel{1}{\rightleftharpoons}} \mathrm{B}_{\mathrm{X}} \underset{2 \mathrm{a}}{\stackrel{2}{\rightleftharpoons}} \mathrm{C}_{X}$ sehr schnell ablaufen, die Reaktionsschritte $\underset{3}{\mathrm{~A}} \underset{1 \mathrm{a}}{\stackrel{1}{\rightleftharpoons}}$ $\mathrm{B}_{\mathrm{Ph}} \underset{3 \mathrm{a}}{\stackrel{{ }^{3}}{\rightleftharpoons}} \mathrm{C}_{\mathrm{Ph}}$ jedoch langsam, sollte sich die gemessene Reaktionsordnung auf die Nachlieferung von Phenolatstrukturen aus A beziehen, die gemessene Aktivierungsenergie auf den Proze $\beta \mathrm{A} \rightarrow \mathrm{C}_{\mathrm{Ph}}$. Die beobachtete Wellenlängenverschiebung bei dieser Naphtholat-Phenolat-Umlagerung kann also aus 


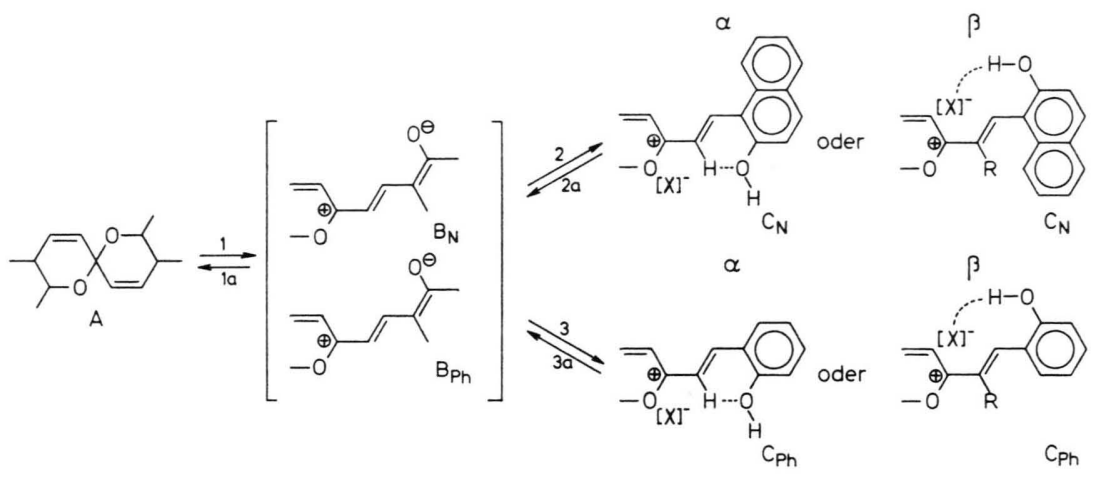

Formel 4

einer Verschiebung des ursprünglich bestehenden Dissoziationsgleichgewichtes heraus erklärt werden.

Zwischen vinylsubstituierten und nicht vinylsubstituierten $o$-Hydroxyarylvinylpyrylium-Salzen wurden vorstehend unterschiedliche $v(\mathrm{C} \cdots \mathrm{O})$-Frequenzen aufgezeigt. Für das Vorliegen einer starken Hybridisierung in nicht vinylsubstituierten $o$-Hydroxyarylvinylpyrylium-Salzen spricht neben der niedrig liegenden $v(\mathrm{C} \cdots \mathrm{O})$-Frequenz die gegenüber vinylsubstituierten $o$-Hydroxyarylvinylpyrylium-Salzen kurzwellig verschobene $v(\mathrm{OH})$ wie auch das kurzwellig verschobene Absorptionsmaximum, aus denen das Vorliegen einer brückengebundenen 6-Ringstruktur C vom Typ $\alpha$ abgeleitet werden kann, obwohl auch das gleichzeitige Vorliegen einer $\beta$-Struktur nicht auszuschließen ist.

Andere Verhältnisse liegen bei vinylsubstituierten Pyryliumsalzen vor. Hier ist durch den Substituenten für C die Ausbildung einer brückengebundenen 6-Ringstruktur vom Typ a nicht mehr möglich, aus sterischen Gründen eine derart weitgehende Hybridisierung wie bei nicht vinylsubstituierten Systemen kaum mehr vorstellbar; die das Verhalten vinylsubstituierter $o$-Hydroxyarylvinylpyryliumsalze charakterisierende Struktur C ist auch der angehobenen $\nu(\mathrm{C}=\mathrm{O})$ zufolge eine ausgeprägtere, lokalisiertere Carboniumstruktur. Beobachtet wird zudem neben einem gegenüber nicht vinylsubstituierten Verbindungen längerwellig verschobenen Absorptionsmaximum eine je nach Substitution mehr oder minder starke Abweichung des Arylvinylrestes von der Planarität. Bei nahezu konstanter Frequenzlage der olefinischen $v(\mathrm{C}=\mathrm{C})$ ist jedoch ein Gang in $v(\mathrm{OH})$ wie auch $v(\mathrm{Cl}-\mathrm{O})$ festzustellen. Dies macht für vinylsubstituierte $o$-Hydroxyarylvinylpyrylium-Salze ein Vorliegen in einer Struktur C vom Typ $\beta$ wahr- scheinlich. Aus den folgenden Moleküldaten sollen die hier gezogenen Schlüsse verdeutlicht werden.

Nachdem das Verhalten der unsubstituierten Grundkörper auf die in Formel 2 skizzierten Umlagerungen zurückgeführt werden konnte, bemühten wir uns nun, das bei einigen vinylsubstituierten Spirobipyranen beobachtete Auftreten verschiedener Absorptionsmaxima aufzuklären. Betrachtet man als Modellsubstanzen symmetrische vinylsubstituierte $o$ Hydroxyarylvinylpyrylium-Salze, bei denen keine Umlagerung der vorher beschriebenen Art möglich ist, so zeigen die in Acetonitril gelösten Pyryliumperchlorate des Spirantyps 8 zunächst die den präparativ hergestellten Farbsalzen entsprechenden Maxima. Eine Bestrahlung unter einer etwa $25 \mathrm{~cm}$ entfernt aufgebauten normalen 60-Watt-Glühlampe über einen längeren Zeitraum hinweg verschiebt das Maximum jedoch kurzwellig, wobei gleichzeitig die zu beobachtende Extinktion abfällt. Anzunehmen ist dabei wieder, daß hier allein das Auftreten der Strukturen B und C wahrscheinlich ist. Bei Berücksichtigung des zur Verschiebung des Absorptionsmaximums ausreichenden Energiebetrags kann nur der Schluß gezogen werden, daß der beobachtete Prozeß der Übergang von Struktur C aus einer anti- in eine $s y n$-Form ist.

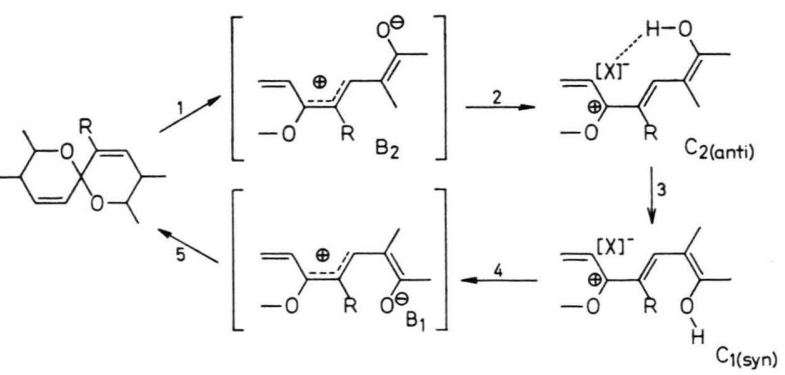

Formel 5 


\begin{tabular}{|c|c|c|c|c|c|c|c|}
\hline \multirow[t]{2}{*}{$\begin{array}{l}\text { Substituent } \\
\mathrm{R}=\end{array}$} & \multirow[t]{2}{*}{$v(\mathrm{OH})$} & \multirow[t]{2}{*}{$v(\mathrm{C}=\mathrm{C})$} & \multirow[t]{2}{*}{$v(\mathrm{C} \cdots \mathrm{O})$} & \multirow[t]{2}{*}{$v(\mathrm{Cl}-\mathrm{O})$} & \multirow[t]{2}{*}{$v(\mathrm{C}-\mathrm{O})$} & \multicolumn{2}{|c|}{$\begin{array}{c}\lambda \quad \text { und } \log \varepsilon \\
\text { der langwelligsten } \\
\text { Bande } 15,16\end{array}$} \\
\hline & & & & & & & \\
\hline \multicolumn{8}{|c|}{$\mathrm{H}-\quad$ nicht dargestellt } \\
\hline$n-C_{5} \mathrm{H}_{11^{-}}$ & 3210 & 1622 & 1544 & 621,1113 & 1036 & 516 & 4,20 \\
\hline $\mathrm{C}_{6} \mathrm{H}_{5^{-}}$ & 3228 & 1622 & 1544 & 624,1112 & 1048,1040 & 528 & 3,38 \\
\hline \multicolumn{8}{|c|}{ 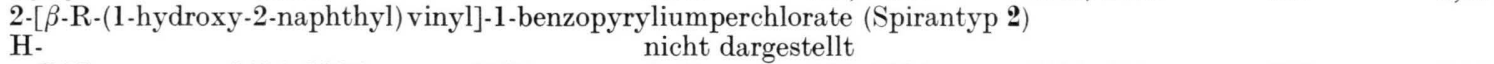 } \\
\hline $\mathrm{n}-\mathrm{C}_{5} \mathrm{H}_{11^{-}}$ & 3415,3225 & 1617 & 1540 & 624,1094 & 1036,1027 & 582 & 3,73 \\
\hline $\mathrm{C}_{6} \mathrm{H}_{5^{-}}$ & 3380,3273 & 1620 & 1540 & 624,1110 & 1042,1031 & 590 & 2,78 \\
\hline \multicolumn{8}{|c|}{ 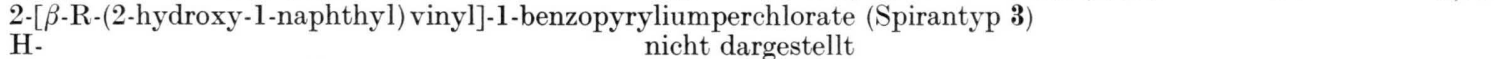 } \\
\hline$n-\mathrm{C}_{5} \mathrm{H}_{11^{-}}$ & 3318 & 1618 & 1535 & 624,1113 & 1037 & 592 & 3,49 \\
\hline $\mathrm{C}_{6} \mathrm{H}_{5^{-}}$ & 3225 & 1619 & 1535 & 625,1109 & 1054,1039 & 600 & 2,50 \\
\hline \multicolumn{8}{|c|}{ 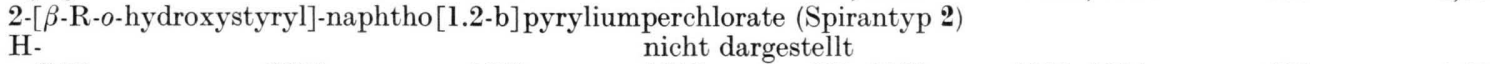 } \\
\hline $\mathrm{n}-\mathrm{C}_{5} \mathrm{H}_{11}-$ & 3215 & 1633 & 1545 & 622,1109 & 1050,1034 & 538 & 4,40 \\
\hline $\mathrm{C}_{6} \mathrm{H}_{5^{-}}$ & 3265 & 1629 & 1541 & 623,1116 & 1052 & 550 & 4,13 \\
\hline \multicolumn{8}{|c|}{$\begin{array}{l}2-[\beta \text {-R-(1-hydroxy-2-naphthyl) vinyl }] \text {-naphtho[1.2] pyryliumperchlorate (Spirantyp 6) } \\
\text { H- }\end{array}$} \\
\hline $\mathrm{n}-\mathrm{C}_{5} \mathrm{H}_{11^{-}}$ & 3190 & 1620 & 1540 & 624,1110 & 1028 & 594 & 4,46 \\
\hline $\mathrm{C}_{6} \mathrm{H}_{5^{-}}$ & 3268 & 1622 & 1537 & 623,1114 & 1026 & 607 & 3,82 \\
\hline \multirow{2}{*}{\multicolumn{8}{|c|}{$\begin{array}{l}2-[\beta \text {-R-(2-hydroxy-1-naphthyl) vinyl }] \text {-naphtho }[1.2 \text {-b }] \text { pyryliumperchlorate (Spirantyp } 7) \\
\text { H- }\end{array}$}} \\
\hline & & & & & & & \\
\hline n- $\mathrm{C}_{5} \mathrm{H}_{11}-$ & 3210 & 1631 & 1539 & 623,1093 & 1047,1036 & 605 & 4,20 \\
\hline $\mathrm{C}_{6} \mathrm{H}_{5^{-}}$ & 3160 & 1616 & 1532 & 624,1114 & 1038 & 620 & 3,25 \\
\hline \multicolumn{8}{|c|}{ 3-[ $\beta$-R-o-hydroxystyryl]-naphtho[2.1-b]pyryliumperchlorate (Spirantyp 3) } \\
\hline $\mathrm{H}-$ & 3303 & 1613 & 1527 & 624,1100 & 1051,1035 & 523 & 4,66 \\
\hline $\mathrm{n}-\mathrm{C}_{5} \mathrm{H}_{11^{-}}$ & 3260,3200 & 1615 & 1548 & 624,1112 & 1050,1040 & 536 & 4,57 \\
\hline $\mathrm{C}_{6} \mathrm{H}_{5-}$ & 3248 & 1614 & 1544 & 624,1111 & 1050 & 544 & 4,40 \\
\hline \multicolumn{8}{|c|}{ 3-[ $\beta$-R-(1-hydroxy-2-naphthyl) vinyl]-naphtho [2.1-b]pyryliumperchlorate (Spirantyp 7 ) } \\
\hline $\mathrm{H}-$ & 3400 & 1606 & 1521 & 621,1115 & 1048 & 574 & 4,65 \\
\hline $\mathrm{n}-\mathrm{C}_{5} \mathrm{H}_{11^{-}}$ & 3300 & 1617 & 1538 & 626,1094 & 1038,1027 & 590 & 4,59 \\
\hline $\mathrm{C}_{6} \mathrm{H}_{5-}^{-}$ & & 1618 & 1539 & 624,1113 & 1035,1027 & 600 & 4,01 \\
\hline \multicolumn{8}{|c|}{ 3-[ $\beta$-R-(2-hydroxy-1-naphthyl) vinyl]-naphtho[2.1-b]pyryliumperchlorate (Spirantyp 8) } \\
\hline $\mathrm{H}-$ & 3235 & 1620 & 1521 & $627,1091,1110$ & 1047,1035 & 584 & 4,65 \\
\hline $\mathrm{n}-\mathrm{C}_{5} \mathrm{H}_{11^{-}}$ & 3162 & 1619 & 1536 & 623,1103 & 1049,1037 & 596 & 4,56 \\
\hline $\mathrm{C}_{6} \mathrm{H}_{5^{-}}$ & 3185 & 1618 & 1535 & 623,1112 & 1048,1035 & 615 & 3,76 \\
\hline
\end{tabular}

Tab. 5. Gang charakteristischer Meßwerte bei vinylsubstituierten $o$-Hydroxyarylvinylpyryliumperchloraten.

Daraus ist zu folgern, daß mit dem Abbau der stabilen $\mathrm{C}_{2}$-Struktur eine der Zyklisierung vorgelagerte $\mathrm{C}_{1}$-Struktur beobachtbar wurde, aus der bei HX-Abspaltung (Reaktionsschritt 4) auch eine andere B-Struktur resultieren sollte. Mit der Beobachtung stimmt überein, daß mit dem Auftreten der $\mathrm{C}_{1}$ Struktur und der damit verbundenen weiteren $\mathrm{Ab}$ weichung von der Planarität auch eine herabgesetzte Übergangswahrscheinlichkeit verbunden sein $\mathrm{mu} \beta$; durch die Aufhebung der Brückenbindung muß zudem die Wahrscheinlichkeit für die Rezyklisierungsschritte $4 / 5$ ansteigen. Die Wellenlängen- bzw. Extinktionsgänge verschiedener Kontrollsubstanzen werden nachfolgend gegeben.

Bemerkenswert ist die enge Verknüpfung der sterischen Verhältnisse des Moleküls mit dem beobachteten Verhalten. Diese allein sollten als Ursache für das Auftreten des zur kurzwelligen Verschiebung des
Absorptionsmaximums führenden Reaktionsschrittes 3 bei den Verbindungen $1-4$ angesehen werden, der bei 5-12 nicht beobachtet werden konnte; ana$\log 1-4$ wäre bei einem langsamen Ablauf des Reaktionsschrittes 3 auch bei den Verbindungen 5-12 eine kurzwellige Verschiebung ihrer Maxima zu erwarten gewesen.
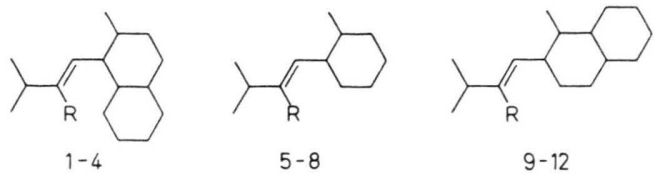

Formel 6

Es wurde oben bereits abgeleitet, daß mit dem Aufheben der durch Brückenbindung stabilisierten anti-Anordnung (Formel 5) die Wahrscheinlichkeit für das Auftreten der Rezyklisierungsschritte 4/5 
o-Hydroxyarylvinylpyrylium-Salz

1) 3-[ $\beta$-n-Amyl-(2-hydroxy-1-naphthyl) vinyl $]$ naphtho[2.1-b] pyryliumperchlorat

2) 3-[ $\beta$-Phenyl-(2-hydroxy-1-naphthyl) vinyl] naphtho[2.1-b] pyryliumperchlorat

3) 2-[ $\beta$-n-Amyl-(2-hydroxy-1-naphthyl) vinyl]-1-benzopyryliumperchlorat

4) 2-[ $\beta$-Phenyl-(2-hydroxy-1-naphthyl) vinyl]-1-benzopyryliumperchlorat

5) 3-[ $\beta$-n-Amyl-o-hydroxystyryl] naphtho[2.1-b]pyryliumperchlorat

6) 3-[ $\beta$-Phenyl-o-hydroxystyryl] naphtho $[2.1-\mathrm{b}]$ pyryliumperchlorat

7) 2 -[ $\beta$-n-Amyl-o-hydroxystyryl]-1-benzopyryliumperchloart

8) 2 -[ $\beta$-Phenyl-o-hydroxystyryl]-1-benzopyryliumperchlorat

9) 2 -[ $\beta$-n-Amyl-(1-hydroxy-2-naphthyl) vinyl] naphtho[1.2-b] pyryliumperchlorat

10) 2-[ $\beta$-Phenyl-(1-hydroxy-2-naphthyl) vinyl] naphtho[1.2-b]pyryliumperchlorat

11) 2-[ $\beta$-n-Amyl-(1-hydroxy-2-naphthyl) vinyl]-1-benzopyryliumperchlorat

12) 2-[ $\beta$-Phenyl-(1-hydroxy-2-naphthyl) vinyl]-1-benzopyryliumperchlorat

Wellenlänge*

Extinktion *

im Hellen im Dunkeln

$\begin{array}{lll}580-565^{130} & \mathrm{sa}^{130} & \mathrm{a}^{150} \\ 593-547^{130} & \mathrm{sa}^{130} & \mathrm{a}^{150} \\ 573-540^{288} & \mathrm{sa}^{288} & \mathrm{a}^{288} \\ 589-535^{192} & \mathrm{sa}^{192} & \mathrm{sa}^{192 * *} \\ 524^{90} & \mathrm{sa}^{90} & \mathrm{a}^{90} \\ 535^{90} & \mathrm{sa}^{90} & \mathrm{a}^{90} \\ 505^{130} & \mathrm{c}^{130} & \mathrm{c}^{150} \\ 515^{130} & \mathrm{c}^{130} & \mathrm{c}^{150} \\ 580^{310} & \mathrm{a}^{310} & \mathrm{c}^{310} \\ 593^{310} & \mathrm{a}^{310} & \mathrm{c}^{310} \\ 566^{286} & \mathrm{a}^{286} & \mathrm{a}^{288} \\ 580^{120} & \mathrm{sa}^{120} & \mathrm{a}^{288}\end{array}$

Tab. 6. Wellenlängen- und Extinktionsgänge verschiedener o-Hydroxyarylvinylpyrylium-Salze in Acetonitril. * Die Indexzahlen geben die Beobachtungszeit in Stdn. an. ${ }^{*}$ Breites Maximum zwischen 535 und $590 \mathrm{~nm}$. sa $=$ stärker abnehmend $(\triangle A$ $>-0,15), \mathrm{a}=$ geringfügig abnehmend $(\triangle A$ bis $-0,15), \mathrm{c}=$ praktisch konstant $(\triangle A<-0,03)$.

durch das Vorliegen der nicht in dem Maße wie die $\mathrm{C}_{2}$-Struktur planar einstellbaren $\mathrm{C}_{1}$-Struktur ansteigen sollte. Wie bei den in Naphtholatstrukturen vorliegenden, sollte jedoch auch bei den in Phenolatstrukturen vorliegenden $o$-Hydroxyarylvinylpyrylium-Salzen zumindest bei Messungen an in Polymeren eingebetteten Substanzen erwartet werden, daß derartige $\mathrm{C}_{1}$-Strukturen noch nachgewiesen werden könnten. Ihr Auftreten wäre gleichfalls etwa $60 \mathrm{~nm}$ kurzwelliger als das der entsprechenden $\mathrm{C}_{2}$ Strukturen zu erwarten. Die sowohl bei Verbindungen der Typen $\mathbf{1}-\mathbf{3}$ wie auch $\mathbf{6 - 8}$ auftretenden Absorptionen im Erwartungsbereich lassen jedoch keine Zuordnung zu phenolischen $\mathrm{C}_{1}$-Strukturen $\mathrm{zu}^{19}$. Anzunehmen ist deshalb, daß die bei $\beta$-Naphtholatstrukturen beobachtete anti-syn-Umlagerung ein durch sterische Hinderung meßbar gewordener Teilschritt der Rezyklisierungsfolge ist, die demnach auf dem Wege $\mathrm{C}_{2} \stackrel{3}{\rightarrow} \mathrm{C}_{1} \stackrel{4}{\rightarrow} \mathrm{B}_{1} \stackrel{5}{\rightarrow}$ A ablaufen sollte.

Beim 2'-Methyl-3 als einziger vinylsubstituierter Verbindung wurde nun, dem Vergleich mit dem Absorptionsmaximum seines $o$-Hydroxyarylvinylpyryliumchlorats zufolge, gleichfalls eine Naphtholat-Phenolat-Umlagerung beobachtet; gemessen wurde hierfür eine Aktivierungsenergie von 13,6 $\pm 2,0 \mathrm{Kcal} /$ Mol. Bei der Verfolgung dieser Umlagerung war jedoch unter der langwelligen Flanke keine der Naphtholatstruktur entsprechende Bande um
$580 \mathrm{~nm}$ festzustellen, zu beobachten war vielmehr am Flankenende eine schwache Bande bei $620 \mathrm{~nm}$. Ihr Auftreten sollte nun so interpretiert werden, daß sich sowohl die Strukturen $B_{1}$ und $B_{2}$ wie auch $B_{2}$ und $C_{2}$ in einem von der Art des Lösungsmittels und dessen Polarität abhängigen Gleichgewicht befinden, das in einer die Weiterreaktion von $\mathrm{B}_{1 / 2}$ einschränkenden Phase (rigid solvent) meßbar zu $\mathrm{B}_{2}$, in einer diese zulassenden Phase (Lösung) jedoch weitgehend zu $\mathrm{C}_{2}$ hin verschoben ist. Das Auftreten der 620-er-Bande und damit einer $\mathrm{B}_{2}$-Struktur beim 2'-Methyl-3 sollte deshalb als Folge einer sterisch leicht behinderten Rezyklisierung von $\mathrm{B}_{1}$-Naphtholatstrukturen angesehen werden, durch die assoziierte $\mathrm{B}_{2}$-Strukturen noch eine Zeit lang neben der Ausbildung der Phenolatstrukturen $\mathrm{C}_{2}$ her beobachtet werden können. Aus dieser Interpretation ergibt sich eine Antwort auf die Frage, warum eine NaphtholatPhenolat-Umlagerung beim 2'-Methyl-3 noch beobachtet wird, beim $2^{\prime}$ - $n$-Amyl-3 nicht mehr: Die Ursache hierfür ist in den durch den Substituenten bedingten sterischen Verhältnissen der $\mathrm{C}_{2}$-Struktur zu erblicken, die die Reaktionsfolge $\mathrm{C}_{2} \rightarrow \mathrm{C}_{1}$ bei einem raumfüllenderen Substituenten stärker behindern bzw. völlig unterbinden. In Analogie zu den Messungen in Lösung wird auch bei UV-Bestrahlung des in Polyvinylacetat unter Zusatz einer halogenierten organischen Verbindung eingebetteten 2'-Methyl-3 neben dem Maximum bei $535 \mathrm{~nm}$ an der langwellig 
deutlich asymmetrischen Flanke ein Bereich mit verringerter Steilheit zwischen etwa 610 und $690 \mathrm{~nm}$ beobachtet. Derart langwellige, jedoch selten klar ausgeprägte Absorptionsmaxima wurden auch bei anderen Spirobipyranen festgestellt ${ }^{19}$. Ihre Extinktionen waren in jedem Falle geringer als bei den den Salzstrukturen C zuzuordnenden Banden.

Legt man dem nunmehr besser überschaubaren Verhalten von Spirobipyranen die von HeiligmanRIM et al. ${ }^{20}$ entwickelten Vorstellungen zugrunde, so sollten sich alle strukturellen Veränderungen auf dem Wege vom Spiran zum $o$-Hydroxyarylvinylpyrylium-Salz und zurück nach dem in Formel 7 dargelegten Schema formulieren lassen. Das nach HEILIGMAN-RIM sowohl bei Ringöffnung zunächst entstehende wie auch dem Ringschluß vorgelagerte cisIsomer (X-Isomer), ,in dem die starke sterische Abstoßung durch die elektrostatische Anziehung zwischen negativ geladenem Sauerstoff- und positiv ge- ladenem -... Kohlenstoffatom aufgewogen würde“ $\left(\mathrm{X}_{1 / 2} s y n\right.$, anti), dürfte im Verlaufe unserer bei Raumtemperatur durchgeführten Messungen kaum beobachtet worden sein. Die Messungen an 3 und seinen Derivaten wie auch an den in Polymere eingebetteten Spirobipyranen ${ }^{19}$ rechtfertigen vielmehr den Schluß, daß lediglich assoziierte Strukturen $\mathrm{B}_{2 \mathrm{a}-\mathrm{d}}$ so langlebig sind, daß ihre Existenz im Spektrum nachgewiesen werden konnte. Alle langwelliger als die den $\mathrm{C}_{2}$-Strukturen entsprechenden Maxima liegenden Absorptionen sollten demnach den verschiedenen aus $\mathrm{B}_{2 \mathrm{a}-\mathrm{d}}$ möglichen Assoziaten zugeordnet werden.

Formel 7 zeigt aber weitergehende Zusammenhänge zwischen Spirantyp und Struktur des aus ihm $z u$ erwartenden $o$-Hydroxyarylvinylpyrylium-Salzes auf. So sind aus cycloalkylsubstituierten Spirobipyranen nur $\mathrm{C}_{2 b}$ - und $\mathrm{C}_{2 \mathrm{c}}$-Strukturen zu erwarten, während monovinylsubstituierte Spirobipyrane zwar for-
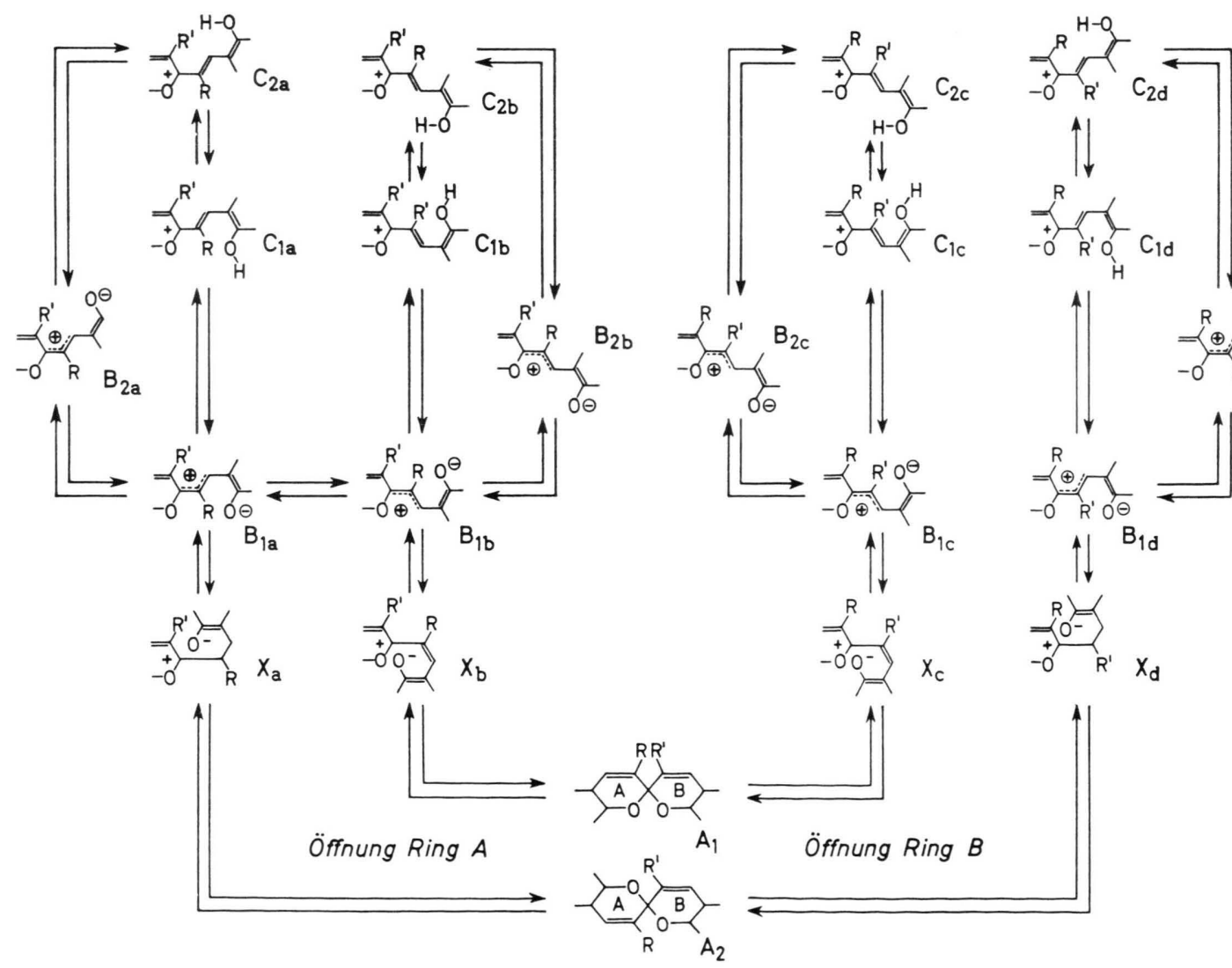

Öffnung Ring $B$
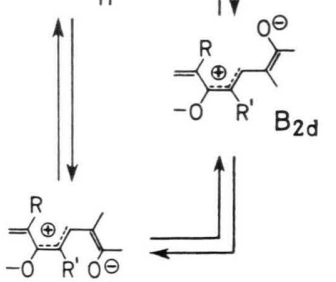

Formel 7

20 R. Heiligman-Rim, Y. Hirshberg u. E. Fischer, J. physic. Chem. 66, 2465, 2470 [1962]. 
mal die Strukturen $\mathrm{C}_{2 \mathrm{a}-\mathrm{d}}$ liefern können, dem Verhalten monovinylsubstituierter Verbindungen der Typen $\mathbf{2}$ und $\mathbf{3}$ zufolge bei diesen jedoch nur die Strukturen $\mathrm{C}_{2 \mathrm{a}}$ und $\mathrm{C}_{2 \mathrm{~b}}$ beobachtet werden. Wie am Beispiel des 2'-Methyl-3 ersichtlich wird, bei dem das Vorliegen einer oder beider Strukturen $\mathrm{C}_{2 \mathrm{c}}$ und $\mathrm{C}_{2 \mathrm{~d}}$ abgeleitet wurde, sollte diese Beschränkung der formal möglichen Strukturen im wesentlichen auf den sterischen Effekt des Vinylsubstituenten zurückzuführen sein. Die den IR-Spektren nach zum Anion hin vorliegende Brückenbindung bei $\mathrm{C}_{2}$-Strukturen ist demzufolge als Auswirkung einer bestimmten räumlichen Anordnung des Moleküls anzusehen. Somit wird die Stabilität von $\mathrm{C}_{2}$-Strukturen ausschließlich durch sterische Fakțoren bestimmt, die auch bei $o$-Hydroxyarylvinylpyrylium-Salzen aus monovinylsubstituierten Spirobipyranen das Vorliegen der Strukturen $\mathrm{C}_{2 \mathrm{c}}$ und $\mathrm{C}_{2 \mathrm{~d}}$ wegen der mit wachsender Größe des Substituenten $\mathrm{R}$ zunehmenden Abdrängung des Anions vom Carboniumkohlenstoff unwahrscheinlich machen. Dieser Schluß sollte Anlaß sein, zu überprüfen, inwieweit verschiedene $R, R^{\prime}$. substituierte, in der Literatur beschriebene Spirobipyrane mit raumerfüllenden Substituenten tatsäch. lich erhalten worden sind.

Beim Auftreten von Thermochromie (Absorptionsmaximum im allgemeinen mit dem des entsprechenden $o$-Hydroxyarylvinylpyrylium-Salzes identisch) ist das Vorliegen der den $\mathrm{C}_{2}$-Strukturen entsprechenden nicht assoziierten $\mathrm{B}_{2}$-Strukturen anzunehmen. Da der Weg von oder zu farbigen Formen des Molekls jedoch generell über die bei der Ringöffnung wie auch beim Ringschluß zu durchlaufenden $\mathrm{B}_{1}$-Strukturen führen sollte, bei denen die Möglichkeit einer trans-cis-Umlagerung des Moleküls in Bezug auf die Anordnung des Vinylsubstituenten des aufgebrochenen Pyranringes zum Sauerstoffatom des erhalten gebliebenen Pyranringes bestehen muß, können also nach keiner Zyklisierung oder Rezyklisierung definierte Spirane der Strukturen $A_{1}$ oder $\mathrm{A}_{2}$ erwartet werden - erwartet werden kann zunächst nur ein Isomerengemisch $A_{1 / 2}$.

\section{Auswertung der Daten von Spirobipyranen}

Bei der Aufnahme der Spektren vergleichbarer, an einem der beiden Pyranringe monoalkylsubstitu-

21 C. Schiele, A. Wilhelm u. G. PaAl, Liebigs Ann. Chem. 722, 162 [1969]

22 Y. YUKAWA, Handbook of organic structural analysis, W. A. Benjamin Inc., New York 1965. ierter asymmetrischer Spirobipyrane ließen sich gewisse Wellenlängenverschiebungen im Bereich der Aromatenabsorption zwischen R- und $\mathrm{R}^{\prime}$-substituierten Spiranen erkennen. Mit den Abb. $2 \mathrm{a}-\mathrm{c}$ werden die Spektren symmetrischer, vinylsubstituierter Spirobipyrane der Typen 1, 6 und 8, mit den Abb. $3 \mathrm{a}-\mathrm{c}$ die Spektren asymmetrischer, vinylsubstituierter Spirobipyrane der Typen 2, 3 und 7 wiedergegeben.

Eine Auswertung der an Spirobipyranen der Typen $\mathbf{1}-\mathbf{3}$ und 6-8 in Dioxan gemessenen Absorptionen ${ }^{21}$ zeigt nun, daß fast alle auftretenden Banden auf das Vorliegen bzw. Dominieren von Absorptionen der in diesen Systemen enthaltenen Grundkörper ${ }^{22}$ zurückgeführt werden können. Eine Bande zwischen 300 und $320 \mathrm{~nm}$ läßt sich jedoch nach diesem Schema nicht mehr zuordnen. Wir vermuten deshalb, daß diese Bande aus einer „Spirokonjugation“ ${ }^{23}$ herrührt. Eine Rechtfertigung für diese $\mathrm{Zu}$ ordnung kann aus der Analyse am Phenylrest substituierter Spirobenzopyrane der Typen 2 und $\mathbf{3}$ abgeleitet werden. Dem IR-Befund nach zeigen hier 6-substituierte Typ-2-Verbindungen beim Übergang von einer Elektronendonator- zu einer Elektronenakzeptor-Substitution einen Anstieg in der Ladungsdichte der Aromat-Pyransauerstoff-Bindung. Parallel dazu ist ein offensichtlich vom mesomeren Effekt des Substituenten abhängiges Abnehmen der Extinktionen der am langwelligsten liegenden Bandentypen 3 und 4 zu beobachten.

Da abgeleitet wurde, daß bei der Zyklisierung der Spirobipyranen zugrunde liegenden $o$-Hydroxyarylvinylpyrylium-Salze immer Isomerengemische der Strukturen $A_{1}$ und $A_{2}$ anfallen sollten, kann daraus gefolgert werden, daß eine aus einer erhöhten Ladungsdichte in der Aromat-Pyransauerstoff-Bindung resultierende "Planarisierung“ der $\mathrm{O}-\mathrm{C}-\mathrm{O}$-Achse oder einer $\mathrm{O}-\mathrm{C}-\mathrm{C}$-Achse (Formel 7) zwangsläufig zu einer gegensinnigen Deformation der $\mathrm{C}-\mathrm{C}-\mathrm{C}$-Achse oder anderen $\mathrm{O}-\mathrm{C}-\mathrm{C}$ Achse und damit zu einer Verminderung der Übergangswahrscheinlichkeit über das Gesamtsystem führen muß. Dasselbe Bild zeigt die Untersuchung 6substituierter Typ-3-Verbindungen, bei 7-substituierten Typ-3-Verbindungen sind die Zusammenhänge nicht so deutlich erkennbar. In Analogie zu Tri-

23 H. E. Simmons u. T. Fununaga, J. Amer. chem. Soc. 89, 5208 [1967]. 

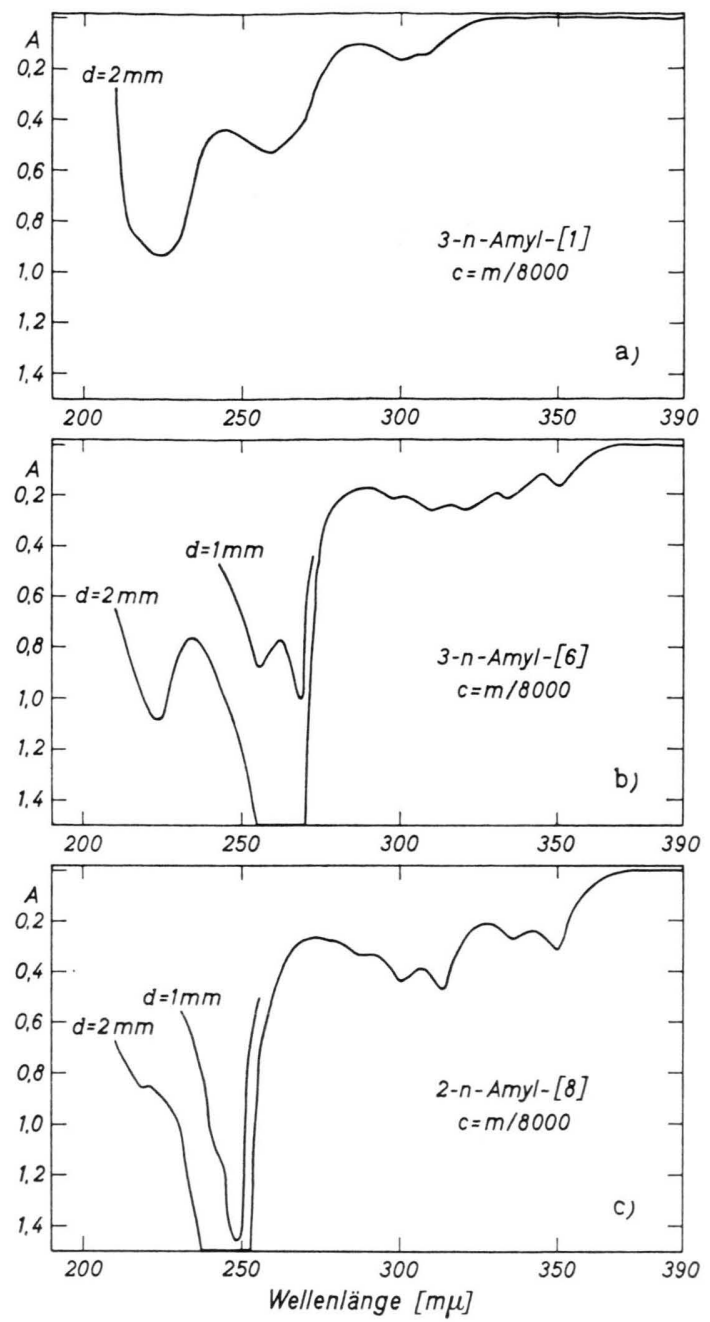

Abb. 2 a. 3-n-Amyl-1; $c=m / 8000$. Teilsystembande 1 a um 258, 2 a um 267, Konjugationsbande 3 um $300 \mathrm{~nm}$.

Abb. 2 b. 3-n-Amyl-6, $c=m / 8000$. Teilsystembande 1 a um 258, 2 a um 267, Konjugationsbanden 3 um 310 und 320 , Gesamtsystembanden 4 um 335 und $350 \mathrm{~nm}$.

Abb. 2 c. 2-n-Amyl-8, $c=m / 8000$. Teilsystembande 1 b um 246, 2 b um 300, Konjugationsbande 3 um 313, Gesamtsystembanden 4 um 335 und $350 \mathrm{~nm}$.
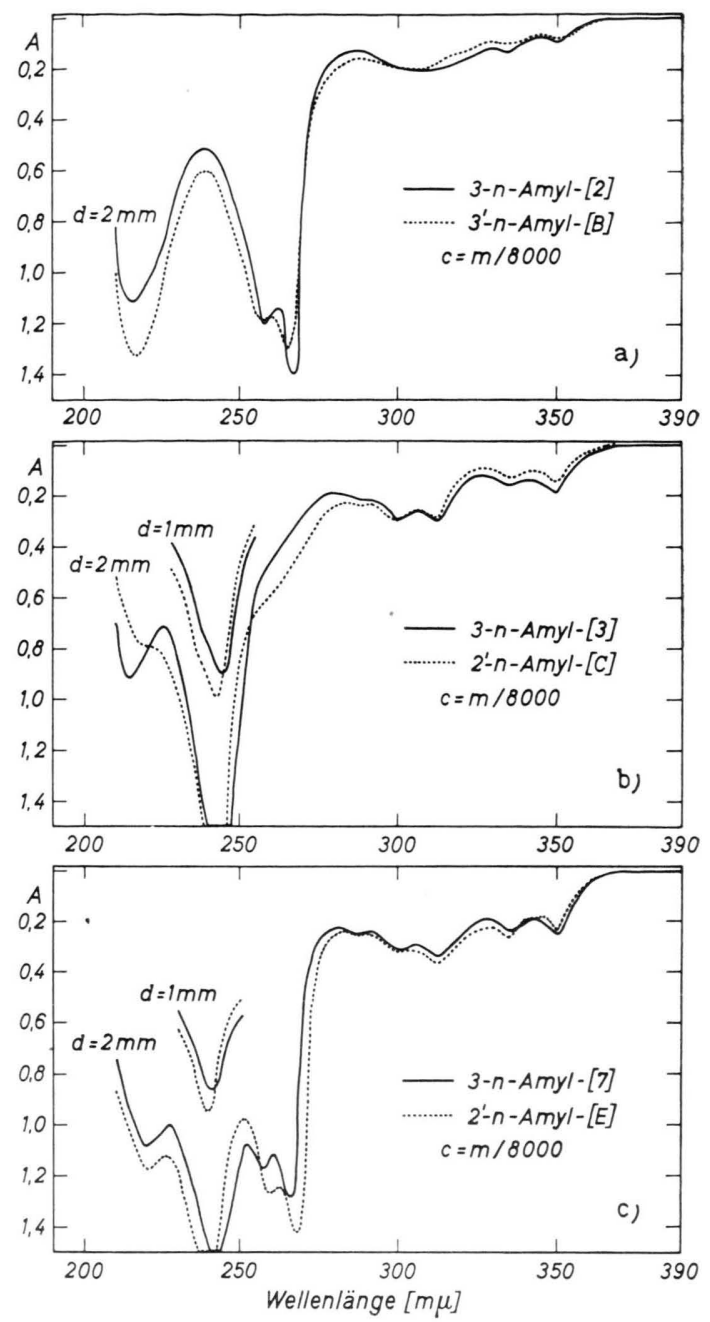

Abb. 3 a. - 3-n-Amyl-2 und - - 3'-n-Amyl-2, $c=m / 8000$. Teilsystembande 1 a um 258, 2 a um 267, Konjugationsbanden 3 um 310 und 320, Gesamtsystembanden 4 um 335 und $350 \mathrm{~nm}$.

Abb. 3 b. - 3-n-Amyl-3 und $\cdots$ 2'-n-Amyl-3, $c=m / 8000$. Teilsystembande 1 b um 246,2 b um 300 , Konjugationsbande 3 um 313, Gesamtsystembanden 4 um 335 und $350 \mathrm{~nm}$.

Abb. 3 c. - 3-n-Amyl-7 und ‥ 2'-n-Amyl-7, $c=m / 8000$. Teilsystembande 1 a um 258,2 a um 267 , Konjugationsbanden 3 um 300 und 313, Gesamtsystembanden 4 um 335 und $350 \mathrm{~nm}$.

Korrektur zu den Abb. $3 \mathrm{a}-\mathrm{c}$ beim Druck: statt B lies 2, statt C lies 3, statt E lies 7 .

einem am Spiro-C-Atom stark verspannten Molekül entsprechenden hochliegenden $v(\mathrm{O}-\mathrm{C}-\mathrm{O})$-Frequenzen gemessen werden; eine deutliche Änderung im Ausmaß der Valenzwinkeldeformation beim Übergang vom Di- zum Trimethylen-Derivat kann erkannt werden. Bemerkenswert ist bei Cycloalkyl- methylindolinospiropyranen ${ }^{8}$ zeigen auch hier die $\mathrm{NO}_{2}$-substituierten Spirobenzopyrane ein aus dem Rahmen fallendes Verhalten.

Eine weitere Auswertung der Spektren zeigt, daß bei den nur in einer $A_{1}$-Struktur möglichen Cycloalkyl-Derivaten der verschiedenen Spirobipyrane die 


\begin{tabular}{|c|c|c|c|c|c|c|c|c|}
\hline \multirow{2}{*}{$\begin{array}{l}\text { Spiran } \\
\text { 3-Methyl-1 }\end{array}$} & \multicolumn{3}{|c|}{$v(\mathrm{C}=\mathrm{C})$} & \multicolumn{2}{|l|}{$v(=\mathrm{C}-\mathrm{O})$} & \multicolumn{2}{|c|}{$v(\mathrm{O}-\mathrm{C}-\mathrm{O})$} & \multirow{2}{*}{$\begin{array}{c}\text { Thermo- } \\
\text { chromie }\end{array}$} \\
\hline & 1666, & 1646 & & 1230 & & 946 & & \\
\hline 3-n-Amyl-1 & & 1641 & & 1234,1224 & & 946, & 938 & - \\
\hline 3-Phenyl-1 & & & 1632 & 1231 & 969 & & & - \\
\hline 2-iso-Propyl-8 & 1653 & 1640 & & 1232 & & 944 & 919 & $(+)$ \\
\hline 2-n-Butyl-8 & 1652, & 1640 & & 1233 & & 943 & 916 & $(+)$ \\
\hline 2-iso-Butyl-8 & & 1643 & & 1234 & 962 & & 917 & $(+)$ \\
\hline 2-n-Amyl-8 & 1650 & 1640 & & 1234 & & 947 & 915 & $(+)$ \\
\hline 2-(3-Methyl) buten $(\Delta 2.3)$ yl-8 & & 1642 & & 1233 & & 946 & 918 & $(+)$ \\
\hline 2-n-Octyl-8 & & 1645 & & 1233 & & 944 & 919 & $(+)$ \\
\hline 2-Phenyl-8 & & & 1631 & 1236 & & & 923 & - \\
\hline 3-n-Amyl-6 & & 1654 & & 1220 & & & 926 & + \\
\hline 3-Phenyl-6 & & & 1647,1643 & 1226 & & 939 & & $(+)$ \\
\hline 3.3'-Dimethylen-1 & 1666 & & & 1214 & 966 & & & - \\
\hline 3.3'-Trimethylen-1 & 1663 & & & 1220 & & 947 & & - \\
\hline 2.2'-Dimethylen- 8 & 1654 & & & 1231 & 966 & & & - \\
\hline $2.2^{\prime}$-Trimethylen-8 & 1661 & & & 1232 & & 948 & & - \\
\hline 3.2'-Dimethylen-3 & $165 \%$ & & & 1219 & 963 & & & - \\
\hline 3.2'-Trimethylen-3 & 1658 & & & 1223 & & 943 & & - \\
\hline 3-Methyl-3 & & 1650 & & 1234 & & 946 & & - \\
\hline $2^{\prime}$-Methyl-3 & 1660, & 1647 & & 1230 & 956, & 943 & & - \\
\hline 3-n-Amyl-3 & & 1645 & & 1234 & & 942 & & - \\
\hline $2^{\prime}$-n-Amyl-3 & 1658 & 1645 & & 1236 & & & 926,921 & $(+)$ \\
\hline 3-Phenyl-3 & & 1646 & 1631 & 1232 & & 945 & & - \\
\hline 2'-Phenyl-3 & & & 1637,1629 & 1231 & & & 935,921 & $(+)$ \\
\hline 3-n-Amyl-2 & & 1650 & & 1240 & & 943 & & - \\
\hline $3^{\prime}$-n-Amyl-2 & & 1654 & & 1240,1228 & & & 935 & + \\
\hline 3-Phenyl-2 & & & 1642 & 1239 & 969, & 961 & & - \\
\hline 3'-Phenyl-2 & & & 1648,1637 & 1229 & & 951 , & 925,910 & + \\
\hline 3-n-Amyl-7 & & 1657 & & 1241 & & 954 , & 929 & + \\
\hline $2^{\prime}$-n-Amyl-7 & & 1658, & 1646 & 1239 & & & 924 & $(+)$ \\
\hline 3-Phenyl-7 & & & 1654 & 1243 & & & 932,909 & $(+)$ \\
\hline 2'-Phenyl-7 & & & 1646,1642 & 1240 & & & 932 & - \\
\hline
\end{tabular}

Tab. 8. Charakteristische IR-Daten am Pyranring substituierter Spirobipyrane.

Derivaten vor allem, daß sie jeweils eindeutige $v(\mathrm{O}-\mathrm{C}-\mathrm{O})$-Absorptionen aufweisen und keine der Verbindungen in siedendem Essigester Thermochromie zeigt.

Aus den in Tab. 8 zusammengefaßten Daten lassen sich nun folgende Schlüsse ziehen:

1. Die unseren bisherigen Befunden und Folgerungen nach in einer $A_{1}$-Struktur vorliegenden, nicht thermochromen Spirobipyrane zeigen eindeutige $v(\mathrm{O}-\mathrm{C}-\mathrm{O})$-Absorptionen. Mit dem Vorliegen einer solchen Struktur scheint zudem eine Lage der $v(\mathrm{O}-\mathrm{C}-\mathrm{O})$ über $940 \mathrm{~cm}^{-1}$ verknüpft zu sein. Langwelliger zu beobachtende $v(\mathrm{O}-\mathrm{C}-\mathrm{O})$-Absorptionen sollten demnach einer $\mathrm{A}_{2}$-Struktur zugeordnet werden.

2. Die in $\mathrm{A}_{2}$-Strukturen vorliegenden Spirobipyrane, bei denen Thermochromie auftreten kann, zeigen eine unterhalb $940 \mathrm{~cm}^{-1}$ auftretende $v(\mathrm{O}-\mathrm{C}-\mathrm{O})$. Deren Lage wird jedoch in Abhängigkeit vom Spirantyp sowohl von der Art der zu einer Deformation der Valenzwinkel am Spiro-C-Atom führenden Sub- stitution an einer der Pyranring-Doppelbindungen wie auch (Tab. 7) von einer zu ElektronendichteÄnderungen im Pyranring führenden Substitution des anellierten Aromaten beeinflußt.

Unsere Messungen an in Polymere eingebetteten Spirobipyranen ${ }^{19}$ ließen dazu eine interessante Korrelation erkennen: Je ausgeprägter eine Verbindung thermochromierte, desto schlechter war der Grad der Umsetzung zum $o$-Hydroxyarylvinylpyrylium-Salz bei UV-Bestrahlung unter Zusatz einer halogenierten organischen Verbindung. Der Grund dafür sollte darin gesehen werden, daß eine intramolekulare Dissoziation durch $k T$ um so wahrscheinlicher ist, je weniger verspannt das Molekül am Spiro-C-Atom vorliegt. Damit steigt aber auch die Wahrscheinlichkeit für eine Rezyklisierung, die bei sehr verspannt vorliegenden Molekülen nurmehr sehr gering sein kann. Daß diese Annahme richtig sein sollte, zeigt der an Verbindungen des Typs $\mathbf{3}$ gefundene $\mathrm{Zu}$ sammenhang zwischen Aktivierungsenergie für den Übergang $A_{2} \rightarrow C_{2}$ und der die Verspannung am 


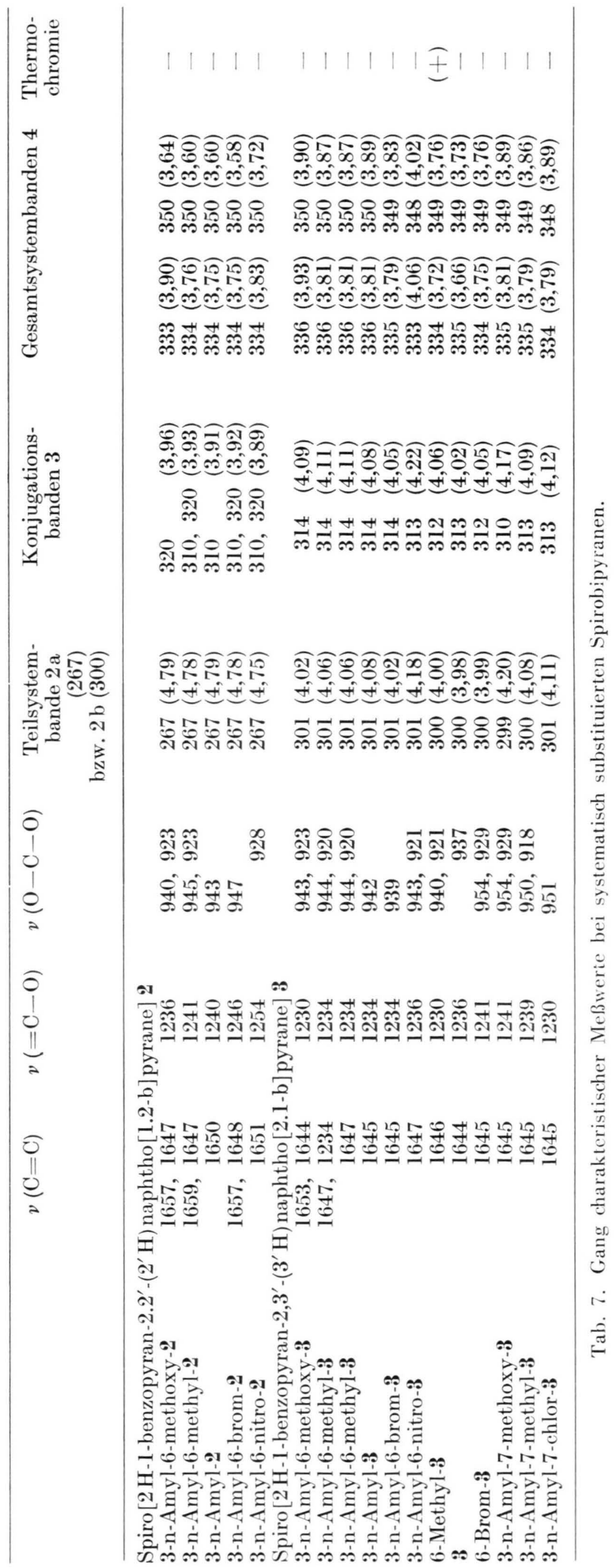

Spiro-C-Atom anzeigenden Lage $\operatorname{der} v(\mathrm{O}-\mathrm{C}-\mathrm{O})$. Lediglich die die niederste $v(\mathrm{O}-\mathrm{C}-\mathrm{O})$ aufweisende Verbindung zeigt Thermochromie in siedendem Essigester.

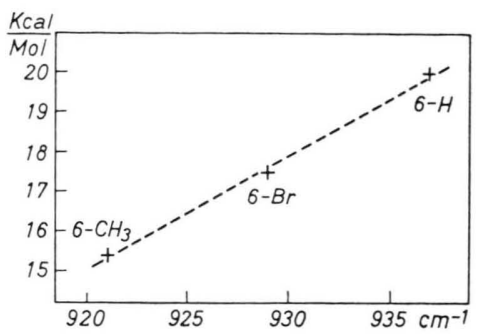

Tab. 9. Zusammenhang zwischen der Aktivierungsenergie für $\mathrm{A}_{2} \rightarrow \mathrm{C}_{2}$ und der Lage $\operatorname{der} v(\mathrm{O}-\mathrm{C}-\mathrm{O})$ bei einigen Spiranen des Typs 3 .

Die Mitarbeit von Herrn G. ARNold, der die zahlreichen, im Verlauf dieser Untersuchung angefallenen IR-Spektren auswertete, sei dankend anerkannt. 